Neurosurg Focus 20 (4):E13, 2006

\title{
Convection-enhanced delivery of immunotoxins and radioisotopes for treatment of malignant gliomas
}

\author{
William A. Vandergrift, M.D., and Sunil J. Patel, M.D. \\ Division of Neurosurgery, Department of Neurosciences, Medical University of South Carolina, \\ Charleston, South Carolina
}

\begin{abstract}
$\checkmark$ The treatment of malignant gliomas has advanced significantly in the past 15 years. The simultaneous development of new targeting agents and techniques to deliver these high-molecular-weight compounds has led to improved efficacy and promising results in Phase III trials. Convection-enhanced delivery (CED) of macromolecules has emerged as the leading delivery technique for the treatment of malignant gliomas. A summary of the basic principles of CED and a review of the current human trials of protein targeting agents are provided.
\end{abstract}

KeY WORDS • convection-enhanced delivery $・$ brain neoplasm $・$ immunotoxin

$\mathrm{D}$ ESPITE advances in chemotherapy, malignant gliomas carry a poor prognosis. Based on data from the Central Brain Tumor Registry of the US, it was predicted that 43,800 new cases of malignant and nonmalignant brain tumors would be diagnosed in 2005.6 Collected data from 1998 to 2002 from this registry show that $40 \%$ of all newly diagnosed brain tumors are gliomas and that $20.3 \%$ are GBMs. Surveillance, Epidemiology, and End Results data from the National Cancer Institute includes reports of 12,760 deaths from brain and other CNS tumors in 2005. ${ }^{36}$

Survival rates for patients with GBM who are treated using the current therapeutic modalities of surgery, radiotherapy, and chemotherapy remain dismal. This longstanding, three-part intervention has had a poor impact on outcome, due in part to the infiltrative nature of malignant gliomas and the protected location in which the tumor cells reside. Resection fails to rid the patient of disease because of the behavior of these tumors and the surgeon's desire not to produce a neurological deficit with this treatment. Radiotherapy focuses on the cancer cell's ability to replicate but unfortunately is limited by its toxicity to the functional but infiltrated surrounding brain tissue. Tumorinfiltrated brain tissue is protected from the systemic circ-

Abbreviations used in this paper: $\mathrm{AA}=$ anaplastic astrocytoma; $\mathrm{BBB}=$ blood-brain barrier; $\mathrm{CED}=$ convection-enhanced delivery; $\mathrm{CNS}=$ central nervous system; GBM = glioblastoma multiforme; $\mathrm{IL}=$ interleukin; $\mathrm{mAb}=$ monoclonal antibody; $\mathrm{MR}=$ magnetic resonance; SPECT = single-photon emission computerized tomography. ulation by the BBB, which prevents most chemotherapeutic agents from effectively reaching the cancer cells. Attempts to deliver chemotherapeutic agents to the brain via intravenous or oral routes have thus been thwarted by high systemic exposure and toxicity.

To overcome systemic effects and achieve high concentrations of drug in tumors, several methods have evolved to deliver local/regional chemotherapeutic agents. These include BBB breakdown, infusions into cerebrospinal fluid, and a variety of intraparenchymal delivery techniques such as bolus injections, slow release using biodegradable vehicles, and convection-enhanced or bulk flow delivery of macromolecules. ${ }^{5}$

\section{Drug Delivery Methods}

\section{Local/Regional Drug Delivery: Bypassing the BBB}

Administration of intravenous chemotherapy following temporary opening of the BBB by using intraarterial mannitol has achieved some success as a treatment for lymphomas. ${ }^{17,30}$ Extrapolation of this technique to other malignant tumors of the CNS has been limited by toxic side effects and the complexity of the procedure. Bolus injection of chemotherapy agents and placement of a chemotherapeutic, drug-impregnated, biodegradable wafer into a tumor resection cavity both rely on the principle of diffusion to drive the drug into the infiltrated brain. ${ }^{5}$ The concentration profile along the diffusion vector from the delivery site shows sharp decrement along its axis, with therapeutic doses of drug often found no more than a few millimeters from the site of highest concentration. This 
restriction in movement is a consequence of the resistance to diffusion in the brain tissue, and unfortunately, it significantly limits the efficacy of this therapy. ${ }^{39}$

\section{Convection-Enhanced Delivery}

In the last decade, CED has emerged as a promising method for the delivery of high concentrations of macromolecules to larger regions of brain tissue. ${ }^{3}$ The general principle of CED involves the stereotactically guided insertion of a small-caliber catheter into the brain parenchyma. Through this catheter, infusate is actively pumped into the brain parenchyma and is pushed mainly through the interstitial space. The infusion is continued for several days and then the catheters are removed at the bedside. In contrast to the millimeter distances obtained with simple diffusion, CED has been shown in laboratory experiments to deliver high-molecular-weight proteins $2 \mathrm{~cm}$ into the brain parenchyma after as little as 2 hours of continuous infusion. This was accomplished without causing cerebral edema and was unaffected by capillary loss or metabolization of the macromolecule. With these initial experiments, CED was established as a viable method for providing regional distribution of molecules as large as immunotoxins and radioisotope-conjugated antibodies, and even some conventional chemotherapeutic agents. ${ }^{2,12,22-25}$ Thus, compared with other therapies, CED offers the advantages of homogeneous exposure of regional brain tissue to high concentrations of chemotherapeutic agents while minimizing systemic and CNS toxicity.

\section{Targeted Therapy}

The ability to deliver a chemotherapeutic agent to the site of resection does not guarantee a good outcome. An ideal therapy should not only provide sufficient delivery of the agent to all of the cancer cells, but it should also differentiate between normal brain cells and cancer cells, being benign to the former and tumoricidal to the latter. $4,8,18,27,40$ Chimeric agents such as radiolabeled antibodies and immunotoxin conjugates have been developed with these principles in mind. These ideal agents are designed so that one part of the macromolecule carries a recognition complex that identifies and binds to the cancer cell, and the other part causes cell death. ${ }^{9}$ Much work continues in the attempt to identify particular cell surface markers and internal proteins that are unique to malignant gliomas. Elucidation of these unique proteins will allow the creation of very specific chimeric chemotherapeutic agents. Examples of proteins that are overexpressed, but are not necessarily unique to malignant gliomas, include the transferrin receptor and IL-13. . $^{10,11,14,15}$ True tumor-specific markers have been harder to identify, and these remain an active area of research interest. 1,16,19,20,26,28,29,31,36,37

The tumoricidal portion of a conjugated targeting agent may take one of many forms. Toxins may generally be divided into the two main categories of bacterial- and plant-derived agents. The most common bacterial toxins include pseudomonas endotoxin and diphtheria toxin, whereas the most common plant toxin is ricin. The wildtype bacterial toxins have essentially two functional subunits. Subunit A is responsible for disruption of protein synthesis, whereas subunit B is responsible for binding and translocation into the cell. To prevent nonspecific cell binding and internalization, subunit B is modified either through deletion or mutation. Identification of the appropriate cell then becomes the function of the conjugated targeting protein or antibody.

Radioisotopes can also be bound to targeting molecules to deliver very localized radiation to malignant gliomas. ${ }^{2}$, ${ }^{32,34}$ One such example is Cotara (Peregrine Pharmaceuticals, Inc., Tustin, CA), which is an ${ }^{131}$ I-labeled chimeric $\mathrm{mAb}$ (also called ${ }^{131} \mathrm{I}$-chTNT-1/B mAb) that is specific for a universal intracellular antigen (that is, DNA/histone H1 complex) exposed in the necrotic core of malignant solid tumors. At first inspection, this does not seem to address the nonnecrotic, migrating portion of the tumor. Experimental evidence demonstrates, however, that the mAb also targets viable tumor cells via what are thought to be leaky nuclear and external cellular membranes. ${ }^{13}$ This antigen provides an abundant, insoluble, nondiffusible anchor for the $\mathrm{mAb}$. Once it is localized to necrotic regions and some living cells of the tumor, Cotara delivers a cytotoxic dose of ${ }^{131} \mathrm{I}$ radiation to the living tumor cells.

\section{Clinical Trials}

Several Phase I and II trials have been completed, and Phase III trials are underway in which immunotoxins or radioisotope-labeled antibodies are being used with CED. Much has been learned about the application of CED to the treatment of malignant gliomas, and investigations are continuing in Phase III trials. We describe here three trials that are representative of this work (these trials are summarized in Table 1).

\section{Cotara Trial}

In a combined single-center Phase I and a multiinstitutional Phase II trial, 51 patients with histologically confirmed recurrent or newly diagnosed malignant gliomas

TABLE 1

Summary of current CED trials

\begin{tabular}{llll}
\hline \hline \multicolumn{1}{c}{ Trial Name } & \multicolumn{1}{c}{ Toxin } & \multicolumn{1}{c}{ Targeting Agent } & \multicolumn{1}{c}{ Trial Phase } \\
\hline Cotara (NABTT-0404) & iodine 131 & chTNT-1/B mAb & I* \\
TransMID (KSB-311R/CIII/001) & diphtheria & transferrin receptor & III; accruing patients \\
PRECISE (IL13-PEI-301-R03) & PE38QQR $\dagger$ & IL-13 & III; awaiting analysis \\
IL13-PEI-106-R01 & PE38QQR $\dagger$ & IL-13 & I \\
\hline
\end{tabular}

* Previous Phase I and II trials have been completed. Cotara investigators are currently reaccumulating patients for dose-escalation analysis and will then initiate Phase III.

$\dagger$ Pseudomonas exotoxin. 


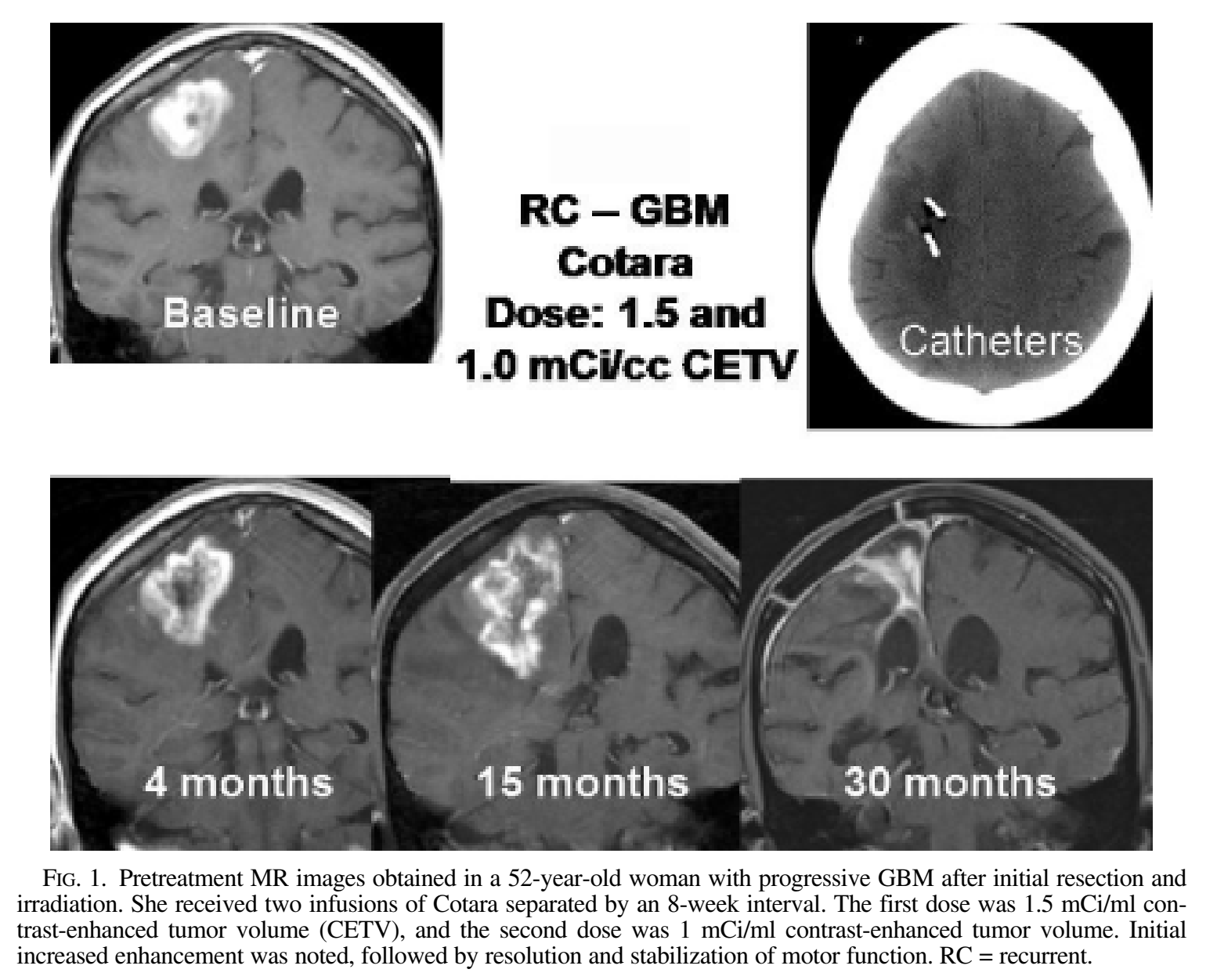

(AA and GBM) received Cotara by CED through stereotactically placed cardiac/peritoneal catheter(s) (Fig. 1)..$^{33}$ Two weeks postinfusion, SPECT scanning was performed to determine the spatial distribution of Cotara. Fifty-one patients, 37 with recurrent GBM, eight with newly diagnosed GBM, and six with recurrent AA, were treated. Of the 37 patients with recurrent GBM, five survived up to 1 year and 2 survived up to 2 years. One patient continues to survive beyond 5 years. None of the patients had a complete radiographically confirmed response, although several had partial responses (Fig. 1). The safety profile showed no systemic effects and only symptomatic cerebral edema at doses above the maximum tolerated dose.

The use of SPECT scanning to observe Cotara distribution in the brain has been helpful. Based on preliminary SPECT studies, it has been suggested that the Cotara infusions provided adequate radiation coverage for the tumor without excessive exposure of surrounding brain tissue. An evaluation of Cotara was done using SPECT scans merged with MR imaging-defined anatomy to examine the effect of drug distribution on survival and to assess treatment outcome parameters (Fig. 2). As might be expected, the longest survival duration posttreatment (in four patients) was consistent with those activity distributions that covered the highest percentage of tumor volume. An analysis in six patients in whom such data could be obtained showed that the correlation between survival and the percentage of tumor coverage was high (nonparametric correlation with Spearman rho test $=0.886, \mathrm{p}=$ $0.019)$ although the demonstration of statistical significance was limited by the small sample size (Fig. 3).

The Cotara study also confirmed that, with small tumors, drug distribution that extended beyond the tumor volume produced longer survival times. This suggests that dose administration to the region outside the enhancing tumor may not be deleterious to clinical outcome and may reflect treatment of the infiltrative portion of a GBM. In future CED trials, a model that considers treatment to a modified "clinical treatment volume" that reflects a reasonable tumor margin and extended distribution of the targeting agent should be factored into the dose administration algorithm. This may include a treatment margin up to several centimeters beyond the baseline, contrast-enhanced, MR imaging-confirmed volume that was established for planning.

Other planning challenges for optimal drug delivery identified during Cotara treatment include antibody binding, local cerebral and tumor architecture, delivery rate, forward pressure of the infusate, catheter placement relationship, and catheter effect on the surrounding tissue. It is likely that solutions to each of these variables will change with the type of infusate used and the conditions in which the catheters are placed. For the small patient cohorts examined in the Cotara trials, these variables, combined with 


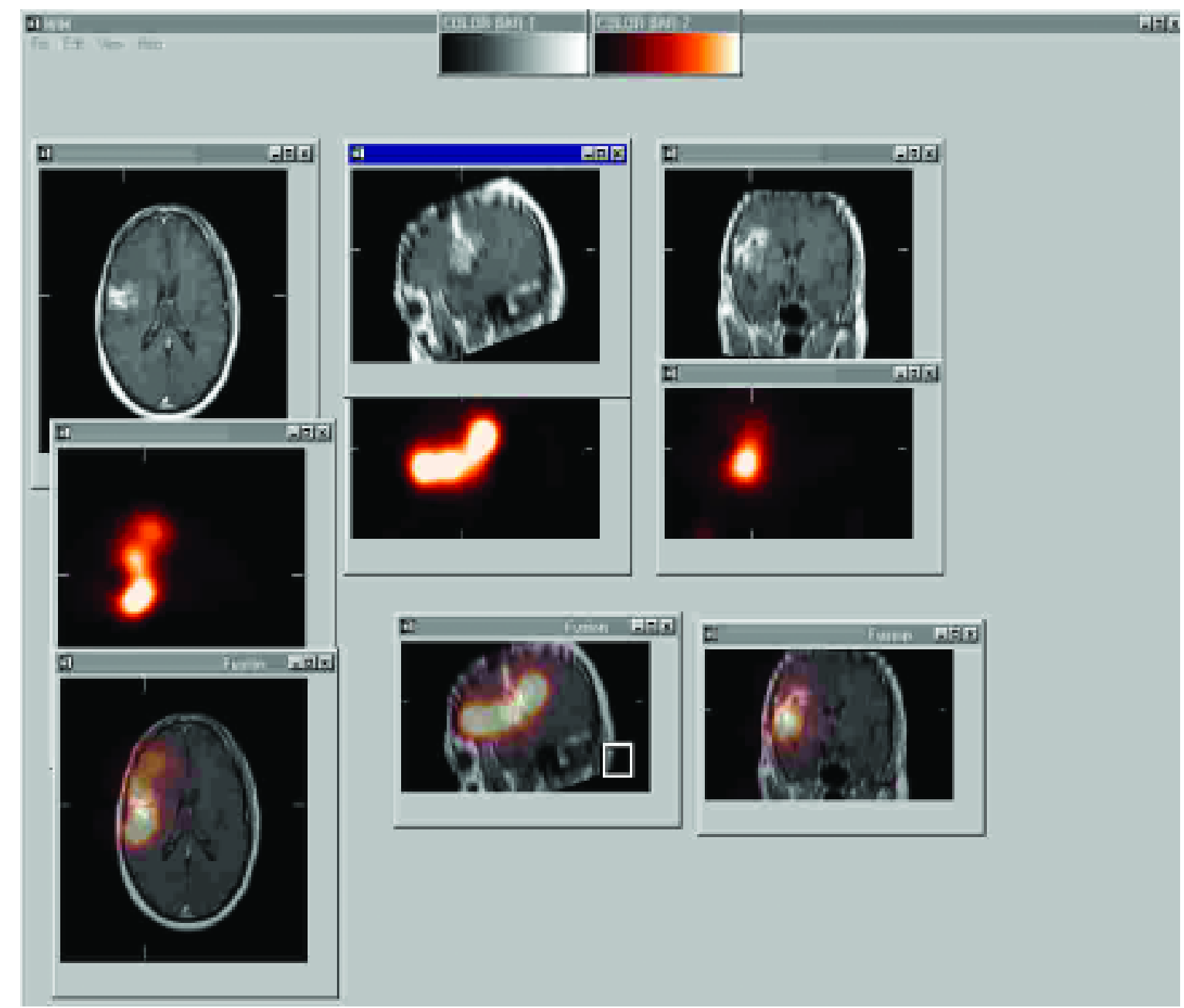

FIG. 2. Neuroimages obtained in a patient with recurrent GBM. The image collage consists of MR (upper row), SPECT alone (center row), and fused images (lower row). Each row contains image projections in axial, sagittal, and coronal views, reading from left to right.

the quantitative study of both activity localization and the resultant absorbed dose delivery, exemplify the complexity of the problem when repeatable and predictable dose delivery is desired for these tumor targets.

Examination of the Cotara data has revealed other useful information about CED in general. The high-dose radiation used in the Cotara trial, although responsible for the tumoricidal effects in malignant gliomas, is also thought to produce an initial increase in enhancing tissue (Fig. 1). This effect has also been seen in several other CED infusions, for which it is speculated that the inflammatory effects from the immunotoxin or radioisotope produce enhancement in the area of the infusion. The enhancement has made differentiation between tumor growth and tumor response difficult to establish using standard MR imaging. Advances in tumor imaging will be needed to clarify the evidence.

\section{TransMID Trial}

The agent TransMID 107 (transferrin-CRM107) is a 140,000 -molecular-weight immunotoxin that is a conjugate of CRM 107 and transferrin. The CRM107 molecule is a modified diphtheria toxin with a single point mutation in its B chain (Phe for Ser 525) that decreases nonspecific binding by 8000 times, whereas its translocation and enzymatic functions are left intact. It is known that trans-

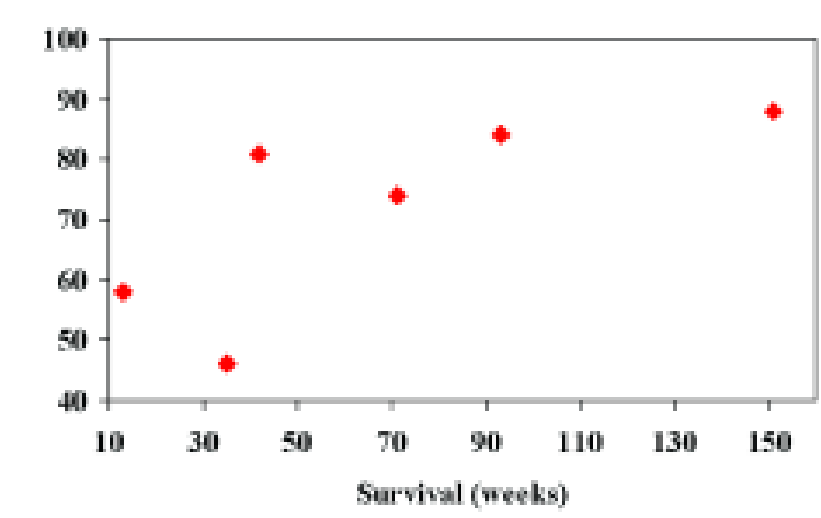

FIG. 3. Graph showing patient survival time in terms of percentage of tumor (contrast-enhanced tumor volume) coverage with Cotara. 


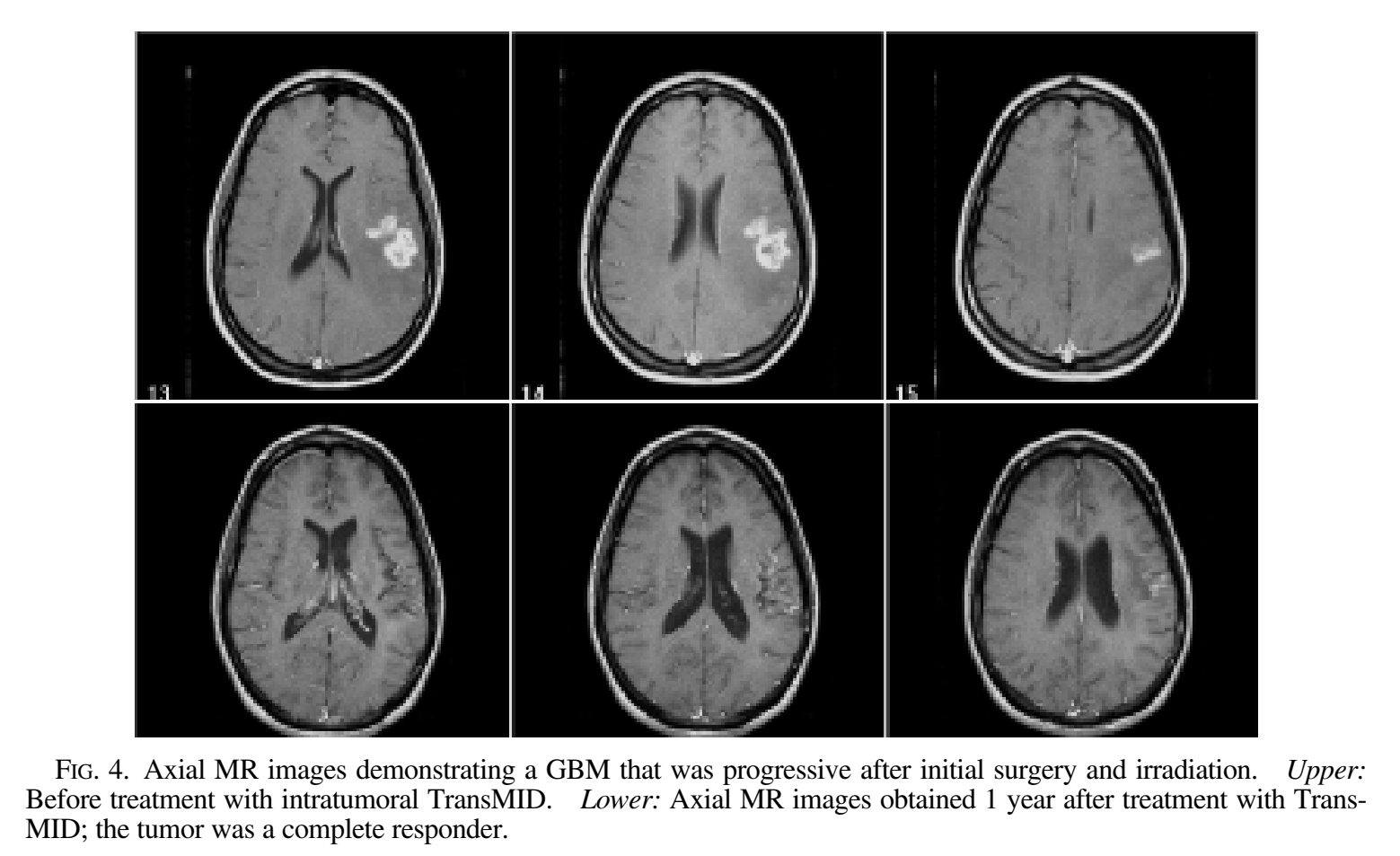

ferrin receptors are expressed on all rapidly dividing cells, including tumors, with minimal expression in nondividing tissues such as normal brain. Therefore, the use of transferrin as a vector or vehicle does not require a unique cellsurface antigen for each type of tumor.

In this study, researchers used transferrin as the vector to target a mutant diphtheria toxin to GBM cells (Patel, unpublished data). The mutation removes the nonspecific binding capability, yet retains the potent translocation and enzymatic functions. The therapeutic conjugate of transferrin and mutated diphtheria toxin was administered by CED with slow infusion via catheters that had been stereotactically placed within the tumor. The Phase I study in 18 patients with malignant CNS lesions conducted by Laske, et al., ${ }^{23}$ at the National Institutes of Health established the safety, efficacy, and suggested dose limits. Total doses more than $28 \mu \mathrm{g}$ and infusate concentrations greater than $0.7 \mu \mathrm{g} / \mathrm{ml}$ produced a higher risk of sustained CNS deficit. This study was the first to show that CED itself is feasible in the treatment of malignant gliomas and that intratumoral infusion of TransMID 107 is safe.

In a Phase II multiinstitutional trial, 33 patients with progressive, recurrent, high-grade gliomas (GBM and AA) were treated with two infusions separated by at least 4 weeks (Patel, unpublished data). Both planned doses were administered to these 33 patients, whereas 11 others received only one dose. Five of the patients had a complete, radiographically confirmed response and seven had a partial response (Figs. 4 and 5). The median survival duration among these responders was 68 weeks, contrasted with 27 weeks among the nonresponders. As shown in Fig. 6, 30\% of the patients were still alive 12 months after the first infusion. The Phase II safety evaluation showed a clinically insignificant elevation of hepatic enzymes in a few cases. Transient cerebral edema was noted in $14 \%$ of the infusions. A Phase III trial is currently underway.

\section{Pseudomonas Exotoxin}

Several trials are either underway or have been completed in which CED of a targeting molecule combined with a pseudomonas exotoxin was used. Sampson, et al. ${ }^{38}$ sought to define the maximum tolerated dose and dose-limiting toxicity of TP-38 delivered by CED in patients with recurrent malignant brain tumors in a Phase I trial. Twenty patients were enrolled in the aforementioned study and received escalating doses. Only one patient experienced Grade IV fatigue at the $100 \mathrm{ng} / \mathrm{ml}$ dose, and the maximum tolerated dose was not established. These investigators did show that tumors in three of 15 patients with residual disease at the time of therapy demonstrated radiographically verified responses. One patient with a complete response survived longer than 83 weeks.

Kunwar ${ }^{21}$ published interim findings from an ongoing Phase I trial in which IL13-PE38QQR, a recombinant mutated pseudomonas exotoxin combined with IL-13, was used. Intratumoral infusion with or without resection was fairly well tolerated when accompanied by corticosteroid prophylaxis, and postresection infusion into the peritumoral brain parenchyma also appeared to be very well tolerated. Although the study was not designed to measure efficacy, Kunwar also noted some improvement in survival. This trial was continued to Phase III testing, in which patients with first recurrences of GBM were treated with either CED of IL13-PE38QQR or Gliadel wafers. The final analysis of 288 patients is expected to be completed in the second quarter of 2006. A Phase I trial is currently underway for IL13-PE38QQR followed by radiation therapy with and without temozolomide. 


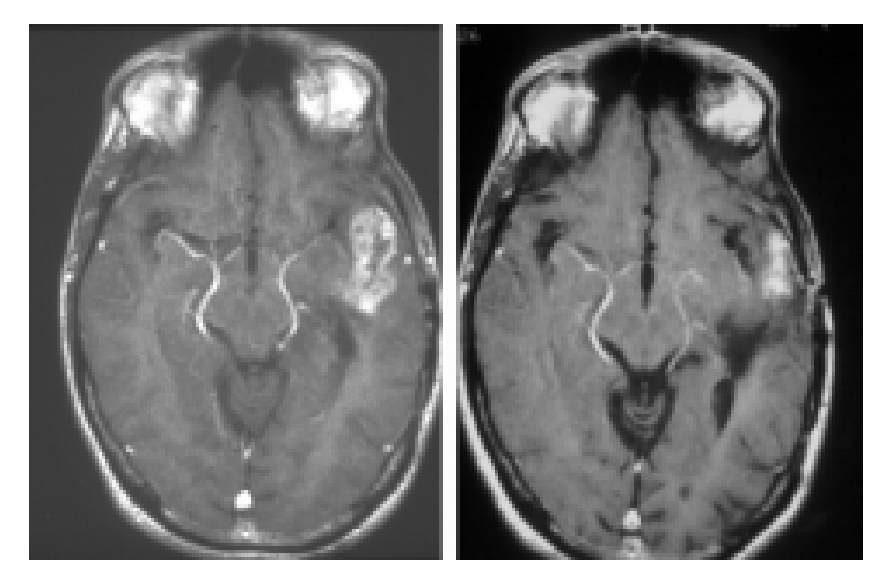

FIG. 5. Axial MR images demonstrating recurrent AA treated with intratumoral TransMID; the tumor was a partial responder. Left: Preinfusion. Right: Six months after treatment with TransMID.

\section{Institutional Experience}

The success of CED relies on recognition of the factors that relate to the placement of the catheters and other infusion parameters that are unique to the process. Significant experimental data had been collected by Laske, et al., ${ }^{22}$ regarding these technical considerations before CED was accepted to human trials. In particular, a variety of factors have been shown to influence the distribution of macromolecules with CED, including rate of infusion, cannula size, concentration of infusate, and preinfusion sealing time. ${ }^{7}$ Postinfusion imaging also has its own set of unique characteristics; the effects of CED can lead to difficulties of interpretation. From our own cumulative experience with 112 infusions, we have developed six guidelines for catheter placement.

\section{Placement of the Catheter}

1) Efficacy of the treatment and patient survival are dependent on the anatomical distribution of drug to the tumor volume. Identification of actual tumor remains unclear even with the most sophisticated imaging. Models are being investigated to determine how to place catheters in locations that will provide the best coverage of tumor. For now it appears that enhancing tissue (which has been confirmed to be tumorous) is the best location in which to place the catheter tip. 2) The tip of the device needs to traverse the longest possible track through the parenchyma to prevent the infusate from tracking back along the catheter and ultimately losing drug into the subarachnoid space. 3) The catheter tip must be placed as far away from any cavity (ventricle, sulcus, or surgical cavity) as possible, so long as it lies within enhancing or tumor-infiltrated tissue. Again, this is to avoid a loss of the infusate into these spaces, because they act as a low-pressure sink. 4) During placement of the device, one should avoid generation of any air pocket around the catheter tip in the parenchyma. The basic principle of CED relies on generation of a high interstitial pressure at the catheter tip. This generates a convective flow from the tip outward into lower-pressure areas. An air pocket delays development of a high interstitial pressure and promotes tracking of infusate back along the catheter. 5) The dynamics of flow with two or more catheters is currently unclear. Investigations in which MR diffusion tensor imaging and mathematical modeling are used should help elucidate these variables so that the catheters may be optimally placed. 6) Each tumor has a unique geometrical configuration and thus a unique map of isopressures and interstitial fluid flow. Convective currents in this situation will depend not only on where the catheter is placed but also on the response of the interstitial pressure vectors and flow patterns unique to each tumor. Current investigations involving dynamic imaging and mathematical modeling during infusion should provide additional understanding of these factors and perhaps improve our ability to distribute agents by using CED.

\section{Postinfusion MR Imaging}

Quite consistent with the effects of the three drugs described earlier is the peculiar increase in enhancement in areas of drug distribution seen after the infusion. Both of the immunotoxins and the radioisotope produced this effect. This increased enhancement adds a level of complexity in MR evaluation after treatment because an increase in enhancement may not necessarily indicate tumor progression. An inflammatory response to the drug is offered as the explanation for this increase in enhancement, although endothelial injury may also play a role. This effect appears to be transient in all cases, often leaving small hypodense areas in the brain. Any trial in which CED is used will need to include frequent, repeated scans following the drug infusion. Both clinical and imaging criteria and, perhaps, histopathological guidelines may be required to establish response to therapy in difficult cases.

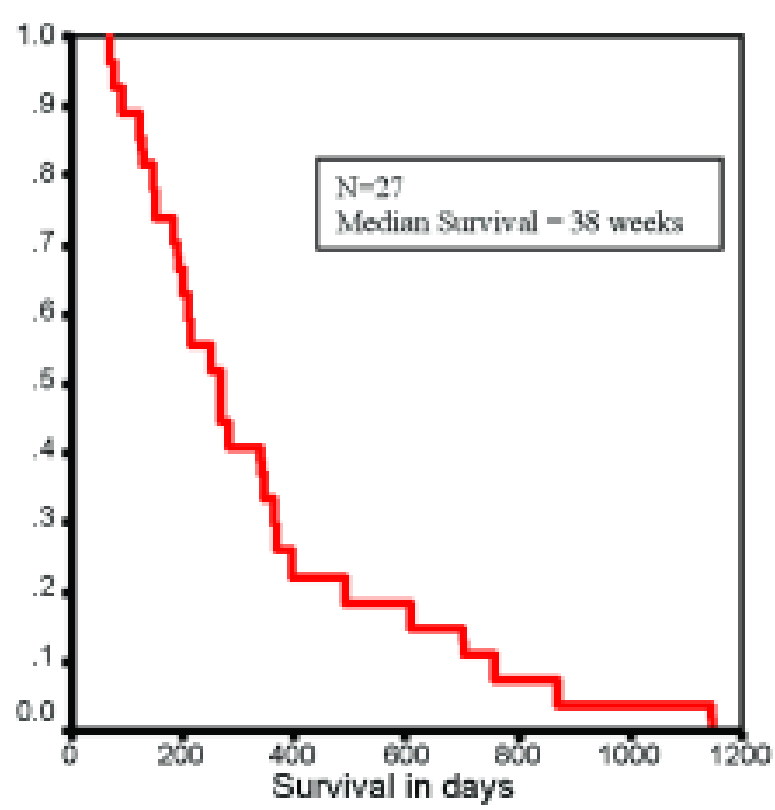

FIG. 6. Graph showing cumulative survival in patients with progressive/recurrent GBM after two doses of TransMID. Numbers on the $\mathrm{y}$ axis designate the percentage of patients surviving. $\mathrm{N}=$ number of patients. 


\section{Limitations of CED}

Although CED has produced promising advances in the treatment of malignant gliomas, it does have some limitations. Foremost is the fact that CED is limited by the efficacy of the targeting proteins and conjoined toxins that it delivers. Malignant gliomas are heterogeneous tumors with molecular diversity. This makes treatment with a single delivery agent incomplete, because currently available molecular targeting agents undoubtedly exclude some cancer cells. Future infusates may include multiple agents, or molecular oncology research may provide different agents that will address this problem.

Although CED allows for wide distribution of macromolecules and is superior to diffusion-based delivery (bolus or biodegradable implants), it has some limitations from a mechanical standpoint. Glioma cells extend well beyond the margins of resection and the contrasted portions observed on neuroimaging. Clinical recurrences of the primary tumor in the adjacent areas and even in the corpus callosum or the contralateral hemisphere attest to this fact. Although CED provides the best volume of distribution of a locally administered drug, it is limited in that a single-sided infusion cannot penetrate bihemispherically. Future research may investigate the effects of widening the regions of catheter placement (perhaps even into the contralateral hemisphere) to address this issue.

Some areas of the brain are inherently difficult to saturate fully with infusate, particularly tumor-infiltrated tissues surrounding a cavity. Such cavities are common in resected tumors, and this difficulty extends to treatment of tissues adjacent to cerebrospinal fluid spaces. These cavities, the particular pressure gradients surrounding tumors, and the natural flow tracts of white and gray matter influence the distribution of infusate. Proper drug delivery will depend on the placement of catheters based on knowledge of these factors. Current understanding of these influential factors continues to evolve with the aid of advances in mathematical modeling and imaging.

\section{Conclusions}

Chemotherapy for malignant gliomas has made much progress in the last 15 years. The options have progressed from high-dose, systemically toxic intravenous chemotherapy to molecular target-based agents convectively delivered as intratumoral infusions, that is, CED. The use of CED has already been shown in multicenter studies to result in improved survival in patients with recurrent GBM, with even a few complete responses. There are many advantages to the use of CED of macromolecules in the brain. This delivery method circumvents the BBB, provides intratumoral and surrounding interstitial drug delivery and high local tumor and peritumoral drug concentrations, and it minimizes systemic exposure. The identification of unique cell surface markers for malignant gliomas should provide the opportunity to develop better targeting agents (immunotoxins and radioisotope-conjugated antibodies) to be administered with CED. Advances in imaging and mathematical models will help to predict the distribution of drug with this technique and consequently can be used to optimize catheter placement.

\section{Disclosure}

This study was supported in part by Peregrine Pharmaceuticals, Inc., Tustin, California, and KS Biomedix, Inc. (which has since been acquired by Xenova Pharmaceuticals, Slough, Berkshire, United Kingdom).

\section{References}

1. Arjona D, Bello MJ, Alonso ME, et al: Molecular analysis of the EGFR gene in astrocytic gliomas: mRNA expression, quantitative-PCR analysis of non-homogeneous gene amplification and DNA sequence alterations. Neuropath Appl Neurobiol 31:384-394, 2005

2. Bigner DD, Brown MT, Friedman AH, et al: Iodine-131labeled antitenascin monoclonal antibody 81C6 treatment of patients with recurrent malignant gliomas: phase I trial results. J Clin Oncol 16:2202-2212, 1998

3. Bobo RH, Laske DW, Akbasak A, et al: Convection-enhanced delivery of macromolecules in the brain. Proc Natl Acad Sci U S A 91:2076-2080, 1994

4. Boskovitz A, Wikstrand C, Kuan C, et al: Monoclonal antibodies for brain tumor treatment. Expert Opin Biol Ther 4: 1453-1471, 2004

5. Brem H, Gabikian P: Biodegradable polymer implants to treat brain tumors. J Control Release 74:63-67, 2001

6. CBTRUS: Statistical Report: Primary Brain Tumors in the United States, 1997-2001. Hunsdale, IL: Central Brain Tumor Registry of the United States, 2004 (http://cbtrus.org/reports// 2004-2005/2005report.pdf)

7. Chen MY, Lonser RR, Morrison PF, et al: Variable affecting convection-enhanced delivery to the striatum: a systematic examination of rate of infusion, cannula size, infusate concentration, and tissue-cannula sealing time. J Neurosurg 90: 315-320, 1999

8. Chiocca EA, Broaddus WC, Gillies GT, et al: Neurosurgical delivery of chemotherapeutics, targeted toxins, genetic and viral therapies in neuro-oncology. J Neurooncol 69:101-117, 2004

9. Debinski W: Local treatment of brain tumors with targeted chimera cytotoxic proteins. Cancer Invest 20:801-809, 2002

10. Debinski W, Gibo DM, Hulet SW, et al: Receptor for interleukin 13 is a marker and therapeutic target for human highgrade gliomas. Clin Cancer Res 5:985-990, 1999

11. Debinski W, Obiri NI, Powers SK, et al: Human glioma cells overexpress receptors for interleukin 13 and are extremely sensitive to a novel chimeric protein composed of interleukin 13 and Pseudomonas exotoxin. Clin Cancer Res 1:1253-1258, 1995

12. Degen JW, Walbridge S, Vortmeyer AO, et al: Safety and efficacy of convection-enhanced delivery of gemcitabine or carboplatin in a malignant glioma model in rats. J Neurosurg 99: 893-898, 2003

13. Epstein A, Khawli L, Chen F, et al: Tumor necrosis imaging and treatment of solid tumors, in Torchillin V (ed): Handbook of Targeted Delivery of Imaging Agents. Boca Raton, FL: CRC Press, 1995, pp 259-288

14. Husain SR, Joshi BH, Puri RK: Interleukin-13 receptor as a unique target for anti-glioblastoma therapy. Int J Cancer 92: 168-175, 2001

15. Husain SR, Puri RK: Interleukin-13 receptor-directed cytotoxin for malignant glioma therapy: from bench to bedside. J Neurooncol 65:37-48, 2003

16. Kawakami K, Kioi M, Liu Q, et al: Evidence that IL-13R $\alpha 2$ chain in human glioma cells is responsible for the antitumor activity mediated by receptor-directed cytotoxin therapy. J Immunother 28:193-202, 2005

17. Kemper EM, Boogerd W, Thius I, et al: Modulation of the blood-brain barrier in oncology: therapeutic opportunities for 
the treatment of brain tumours? Cancer Treat Rev 30: 415-423, 2004

18. Kesari S, Ramakrishna N, Sauvageot C, et al: Targeted molecular therapy of malignant gliomas. Curr Neurol Neurosci Rep 5:186-197, 2005

19. Komata T, Kanzawa T, Kondo Y, et al: Telomerase as a therapeutic target for malignant gliomas. Oncogene 21:656-663, 2002

20. Kuan CT, Wikstrand CJ, Bigner DD, et al: EGFRvIII as a promising target for antibody-based brain tumor therapy. Brain Tumor Pathol 17:71-78, 2000

21. Kunwar S: Convection enhanced delivery of IL13-PE38QQR for treatment of recurrent malignant glioma: presentation of interim findings from ongoing phase 1 studies. Acta Neurochir Suppl 88:105-111, 2003

22. Laske DW, Ilercil O, Akbasak A, et al: Efficacy of direct intratumoral therapy with targeted protein toxins for solid human gliomas in nude mice. J Neurosurg 80:520-526, 1994

23. Laske DW, Youle RJ, Oldfield EH: Tumor regression with regional distribution of the targeted toxin TF-CRM107 in patients with malignant brain tumors. Nat Med 3:1362-1368, 1997

24. Lidar Z, Mardor Y, Jonas T, et al: Convection-enhanced delivery of paclitaxel for the treatment of recurrent malignant glioma: a phase I/II clinical study. J Neurosurg 100:472-479, 2004

25. Lieberman DM, Laske DW, Morrison PF, et al: Convectionenhanced distribution of large molecules in gray matter during interstitial drug infusion. J Neurosurg 82:1021-1029, 1995

26. Liu TF, Tatter SB, Willingham MC, et al: Growth factor receptor expression varies among high-grade gliomas and normal brain: epidermal growth factor receptor has excellent properties for interstitial fusion protein therapy. Mol Cancer Ther 2: 783-787, 2003

27. Lonardi S, Tosoni A, Brandes AA: Adjuvant chemotherapy in the treatment of high grade gliomas. Cancer Treat Rev 31: 79-89, 2005

28. Masi A, Becchetti A, Restano-Cassulini R, et al: hERGI channels are overexpressed in glioblastoma multiforme and modulate VEGF secretion in glioblastoma cell lines. Br J Cancer 93:781-792, 2005

29. Muñoz M, Rosso M, Pérez A, et al: The NK1 receptor is involved in the anti-tumoral action of L-733,060 and in the mitogenic action of substance $\mathrm{P}$ on neuroblastoma and glioma cell lines. Neuropeptides 39:427-432, 2005

30. Neuwelt EA, Goldman DL, Dahlborg SA, et al: Primary CNS lymphoma treated with osmotic blood-brain barrier disruption: prolonged survival and preservation of cognitive function. $\mathbf{J}$ Clin Oncol 9:1580-1590, 1991

31. Newton HB: Molecular neuro-oncology and development of targeted therapeutic strategies for brain tumors. Part 2: PI3K/ Akt/PTEN, mTOR, SHH/PTCH and angiogenesis. Expert Rev Anticancer Ther 4:105-128, 2004

32. Patel S, Breneman JC, Warnick RE, et al: Permanent iodine-125 interstitial implants for the treatment of recurrent glioblastoma multiforme. Neurosurgery 46:1123-1130, 2000

33. Patel SJ, Shapiro WR, Laske DW, et al: Safety and feasibility of convection-enhanced delivery of Cotara for the treatment of malignant glioma: initial experience in 51 patients. Neurosurgery 56:1243-1253, 2005

34. Quang TS, Brady LW: Radioimmunotherapy as a novel treatment regimen: ${ }^{125}$ I-labeled monoclonal antibody 425 in the treatment of high-grade brain gliomas. Int J Radiat Oncol Biol Phys 58:972-975, 2004

35. Raizer JJ: HER1/EGFR tyrosine kinase inhibitors for the treatment of glioblastoma multiforme. J Neurooncol 74:77-86, 2005

36. Ries LAG, Eisner MP, Kosary CL, et al: SEER Cancer Statistics Review, 1975-2002. Bethesda, MD: National Cancer Institute, 2005 (http://seer.cancer.gov/csr/1975_2002/) [Accessed 27 March 2006]

37. Rustamzadeh E, Li C, Doumbia S, et al: Targeting the overexpressed urokinase-type plasminogen activator receptor on glioblastoma multiforme. J Neurooncol 65:63-75, 2003

38. Sampson JH, Akabani G, Archer GE, et al: Progress report of a Phase I study of the intracerebral microinfusion of a chimeric protein composed of transforming growth factor (TGF)- $\alpha$ and a mutated form of the Pseudomonas exotoxin termed PE-38 (TP38 ) for the treatment of malignant brain tumors. J Neurooncol 65:27-35, 2003

39. Subach BR, Witham TF, Kondziolka D, et al: Morbidity and survival after 1, 3-bis(2-chloroethyl)-1-nitrosourea wafer implantation for recurrent glioblastoma: a retrospective casematched cohort series. Neurosurgery 45:17-23, 1999

40. Westphal M, Black PM: Perspectives of cellular and molecular neurosurgery. J Neurooncol 70:255-269, 2004

Manuscript received January 17, 2006.

Accepted in final form March 16, 2006.

Address reprint requests to: Sunil J. Patel, M.D., 96 Jonathan Lucas Street, Suite 428, Charleston, South Carolina 29425. email: patels@musc.edu. 